\title{
Evaluación de un sistema de comunicación Li-Fi aplicado sobre una planta de control
}

Evaluation of Li-Fi communication system applied to a control plant

Jorge Luis Paucar Samaniego ${ }^{1}$, Oswaldo Geovanny Martínez Guashima ${ }^{2}$, Daysi Margoth Guanga Chunata. ${ }^{3} \&$ Fausto Francisco Navarrete Chávez ${ }^{4}$

\begin{abstract}
.
DOI: https://doi.org/10.33262/cienciadigital.v3i3.2.1.784

The document presents the development and implementation of a data transmission system developed with Li-Fi technology to monitor variables of a laboratory plant. In its first stage, an acquisition and processing data is developed to then implement a monitoring system on the web. Virtual instrumentation of acquisition and control is used which was programmed in LabView2018 and the variables that are studied are speed and temperature. Tests on the algorithms were performed, the criteria of integral of the absolute error (IAE) is applied. The integral of the time for the absolute error (ITAE) is used in order to monitor the processes on the web with the Web Publishing Tool and the efficiency of data transmission was contrasted with the Wi-Fi communication. During the tests, the communications data traffic was captured by the Wireshark software and then evaluated by the Steelcentral Packet Analyzer software. As a result, it was possible to determine that Li-Fi has a lower latency time than Wi$\mathrm{Fi}$, what directly influences in the ITAE of the processes.
\end{abstract}

Keywords: Instrumentation Electronic, telecommunications, Mathematics evaluation method, networks, Li-Fi

\footnotetext{
${ }^{1}$ Escuela Superior Politécnica de Chimborazo, carrera de Electrónica y Automatización, Facultad de Informática y Electrónica , Riobamba, Ecuador, jlpaucar@espoch.edu.ec

${ }^{2}$ Escuela Superior Politécnica de Chimborazo, carrera de Telecomunicaciones, Facultad de Informática y Electrónica, Riobamba, Ecuador, geovanny.martinez@espoch.edu.ec

3 Escuela Superior Politécnica de Chimborazo, carrera de Telecomunicaciones, Facultad de Ciencias. Riobamba, Ecuador. daysi.guanga@espoch.edu.ec

${ }^{4}$ Escuela Superior Politécnica de Chimborazo, carrera de Física, Facultad de Ciencias. Riobamba, Ecuador. fausto.navarrete@espoch.edu.ec
} 


\section{Resumen.}

El documento presenta el desarrollo y puesta en marcha de un sistema de transmisión de datos desarrollado con tecnología Li-Fi para realizar un monitoreo de variables de una planta de laboratorio. En su primera etapa se desarrolla una fase de adquisición y procesamiento de datos para luego implementar un sistema de monitoreo en la web. Se utiliza instrumentación virtual de adquisición y control la cual fue programada en LabView2018 y las variables que se intervienen son velocidad y temperatura. Se realizaron pruebas sobre los algoritmos, se emplea los criterios de integral del error absoluto (IAE). La integral del tiempo por el error absoluto (ITAE) es utilizada para el monitoreo de los procesos en la web con la herramienta Web Publishing Tool y se contrastó la eficiencia de transmisión de datos con comunicación Wi-Fi. Durante las pruebas, el tráfico de datos de las comunicaciones fue capturado con el software Wireshark y para luego evaluarlo con el software Steelcentral Packet Analyzer. Como resultado se logró determinar que Li-Fi tiene un tiempo de latencia menor que el de Wi-Fi lo que influye directamente en el ITAE de los procesos.

Palabras claves: Instrumentación electrónica, telecomunicaciones, métodos de evaluación matemáticos, redes, $\mathrm{Li}-\mathrm{Fi}$

\section{Introducción.}

Este documento define el procedimiento y los resultados obtenidos en el desarrollo de transmisión de datos de un proceso de control con comunicación Li-Fi. Más de medio millón de dispositivos son añadidos por año a redes de transmisión de datos a través redes de comunicación inalámbricas, las cuales, son limitadas en gran medida debido a su ancho de banda. Nuevas tecnologías son diseñadas y probadas para suplir las necesidades de comunicación que serán demandadas en pocos años, en contra parte, el espectro visible de luz ofrece un ancho de banda de frecuencia superior, es por eso que, entre una de estas tecnologías que utiliza la luz visible como medio de comunicación está la denominada Li-Fi (Light Fidelity) la cual posee características inalámbricas, bidireccionales, multi usuario y su grado de contaminación electromagnética es prácticamente nulo, por lo cual Li-Fi es un potencial candidato para aplicaciones de comunicación entre dispositivos. La flexibilidad de las redes inalámbricas nos permite establecer ventajas como hardware de tamaño reducido y robustez ante perturbaciones externas [1]. De este modo el estudio tiene la intencionalidad de relacionar el control de procesos con Li-Fi como medio de comunicación para la transmisión de datos de manera bidireccional.

\section{Trabajos Relacionados}

El país en el cual se realizan la mayor cantidad de investigación sobre Li-Fi es Reino Unido, en Latinoamérica no se registra investigaciones al respecto, los trabajos conexos que se 
analizan para iniciar el estudio son los siguientes: [2] establece un análisis de comunicación con base a luz visible, considera que Li-Fi es una aplicación superior a VLC, su uso se enfoca en realizar sistemas inalámbricos completamente conectados a la red, puede ser empleado también en sistemas del tipo IoT, [3] concluye que Li-Fi logra transmisión y recepción de datos y puede resultar más barato debido a la gran cantidad de bombillas desplegadas al interior de edificaciones.[4] realiza una investigación empírica sobre la orientación aleatoria con base en Laplace que puede seguir un usuario aleatorio con el fin de evaluar el rendimiento del sistema. No se puede determinar trabajos que relacionen el control de procesos industriales con Li-Fi como medio de comunicación para la transmisión de datos de manera bidireccional. Las investigaciones antes mencionadas manejan términos generales que son:

Redes Inalámbricas: Las redes sin cableado son más prácticas para los usuarios y permiten desarrollar nuevos productos y servicios, actualmente varias tecnologías son desarrolladas para implementar enlaces punto a punto y multipunto, bajo este concepto se incluye a Li-Fi. Wi-Fi es, hoy en día, la tecnología más utilizada con base en el número de dispositivos que la usan para conectarse a Internet. Los estándares inalámbricos IEEE 802.11a, 802.11b, $802.11 \mathrm{~g}$ y $802.11 \mathrm{n}$ se denominan en conjunto tecnologías Wi-Fi.

Comunicación por luz visible: al hablar de comunicación por luz visible o VLC, se hace referencia a una fuente de iluminación como un LED (diodo emisor de luz) que también es capaz de transmitir información, es decir que VLC es la suma de iluminación más comunicación. Todo esto es factible gracias a su alta velocidad de transmisión de datos. [5] VLC es una tecnología de corto alcance debido a la distancia de propagación de luz que emiten los diodos LED, el espectro de luz visible es modulado para transmitir la información. Como se aprecia en la figura 1, el espectro electromagnético de luz visible cubre entre 350 $\mathrm{nm}$ y $800 \mathrm{~nm}$ de longitud de onda y las frecuencias están comprendidas entre $400 \mathrm{THz}$ y 700 $\mathrm{THz}[6]$.

Figura 1 Espectro Electromagnético

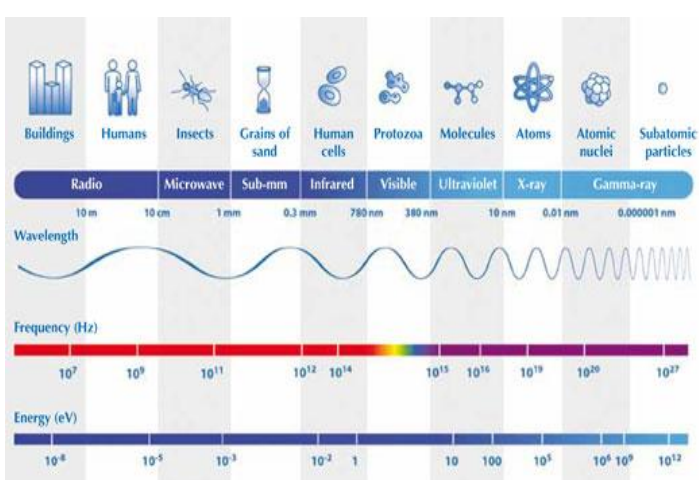

Fuente: https://www.scienceinschool.org 
Light Fidelity: En el estándar IEEE 802.15.7 se encuentra Li-Fi que es una aplicación de las comunicaciones por luz visible (VLC), es decir, usa el espectro de la luz visible para trasmitir datos. Su capacidad es 10.000 veces mayor a la disponible actualmente en el espectro radioeléctrico, una de las ventajas de esta tecnología es que los actuadores como emisores de luz se pueden instalar en lugares cerrados. Li-Fi aprovecha la frecuencia de conmutación de los LED la cual es muy alta esto es otra ventaja que se maneja para trasmitir datos en formato binario. [3]

\section{Metodologia.}

Se diseña un experimento ejecutado sobre un proceso en una planta de control de laboratorio, se desarrolla una transmisión de datos a través de una red Li-Fi y una red Wi-Fi para luego validar los resultados. Se sigue el esquema mostrado en el diagrama de flujo de la figura 2.

Figura 2 Diagrama de flujo de la metodología aplicada

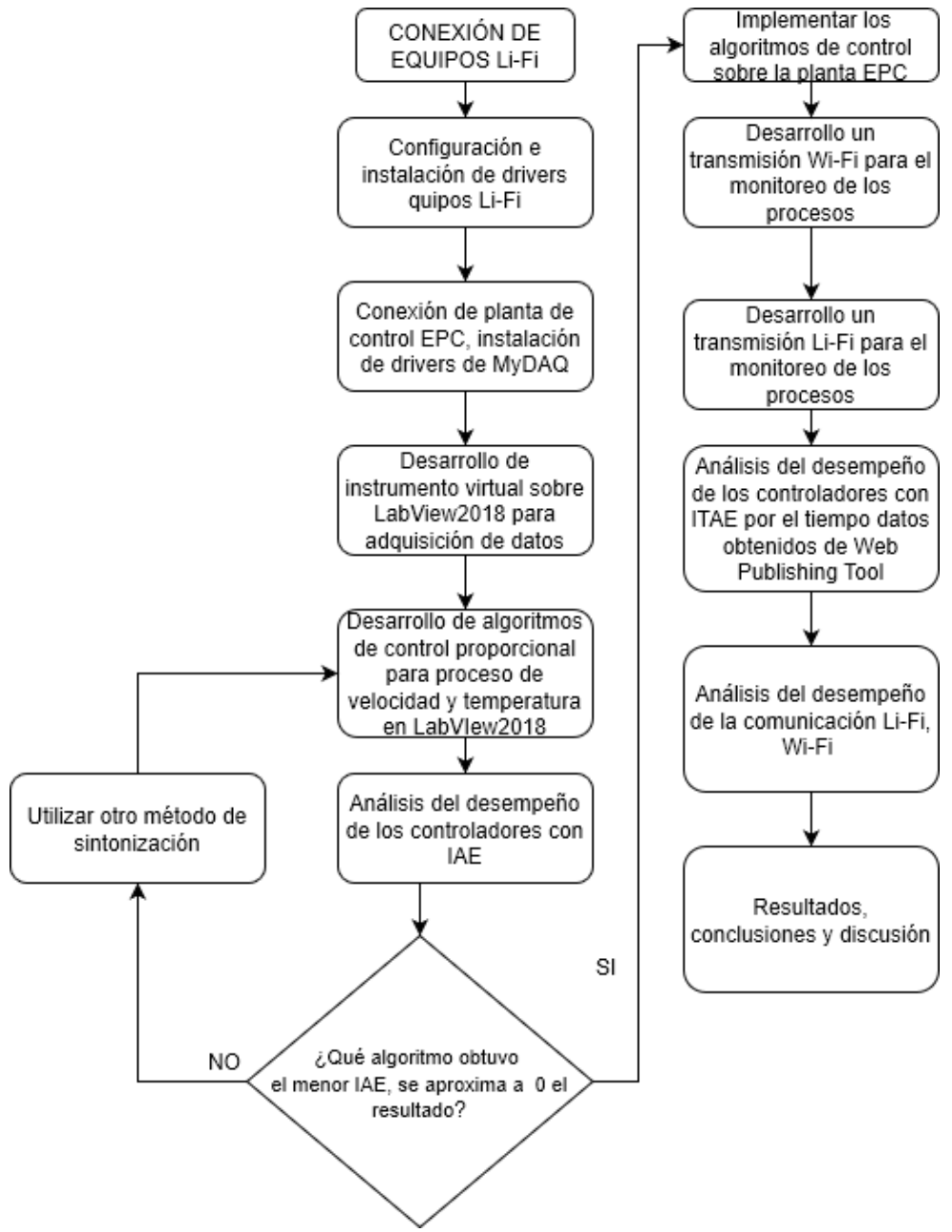

Fuente: Elaboración propia 
En la figura 3 se muestra la arquitectura general del sistema, el primer paso es la adquisición de datos generados en la planta EPC, para lo cual se utiliza una tarjeta de adquisición de datos MyDAQ de National Instruments @ que se comunica a la PC por medio del puerto serial para luego ser procesados y controlados por un instrumento virtual programado sobre la PC. A continuación, los datos son publicados a internet mediante módulos transmisores. Finalmente, los valores de las variables del proceso pueden ser visualizados y monitoreados remotamente a través de una página web para su posterior evaluación.

Figura 3 Arquitectura general del sistema

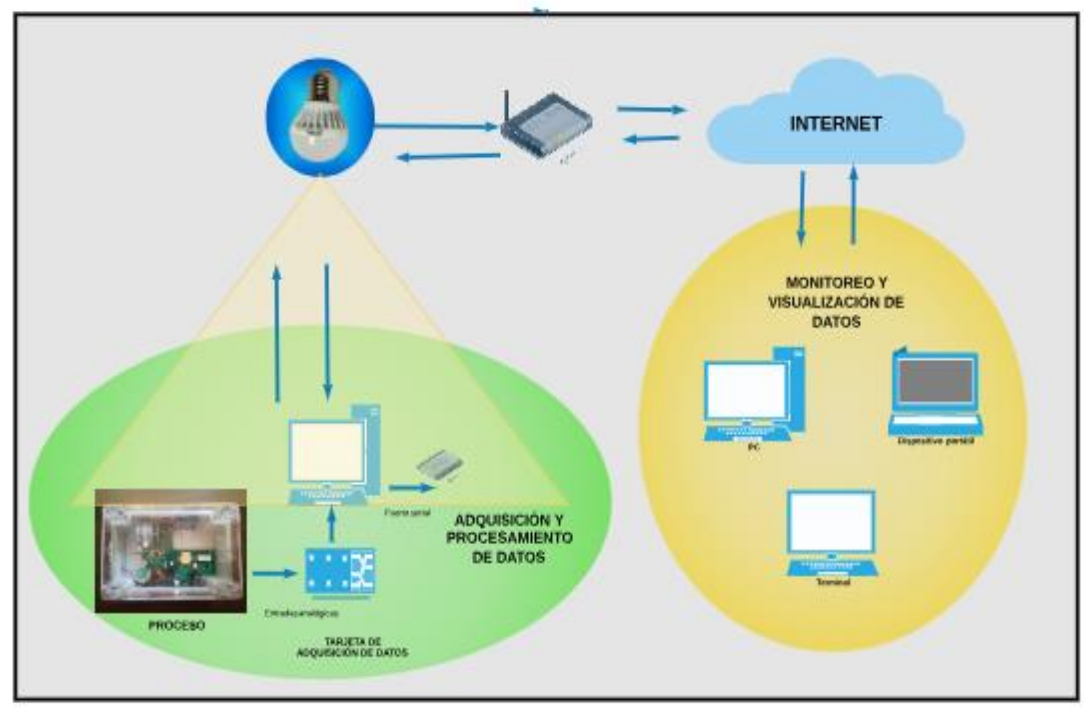

Fuente: Elaboración propia

\section{a) Conexión y Configuración Equipos Li-Fi}

Pocas son las empresas que ofrecen tecnología Li-Fi al usuario final debido a que estos productos están en fase de experimentación y prueba. Se determina que dos empresas a nivel mundial se encuentran en la capacidad de proporcionar estos dispositivos a institutos de investigación, una de ellas es la empresa denominada PURE Li-Fi la cual ofrece varios dispositivos de comunicación y actuadores (lámparas) como se observa en la figura 3, las características principales de los equipos según el fabricante son:

- El sistema Li-Fi totalmente conectado a la red proporciona IPv4 de extremo a extremo y conectividad IPv6.

- Enlace inalámbrico full dúplex que proporciona 43 Mbps tanto de subida como de descarga.

- Distancia mínima de operación $60 \mathrm{~cm}$.

- Distancia máxima de operación 6 m. 
- Punto de acceso admite hasta 8 estaciones.

- Gran cantidad de actuadores luminarias LED.

- Soporta DALI, 0-10 V y CoAP

Figura 4 Conexión dispositivos Li-Fi

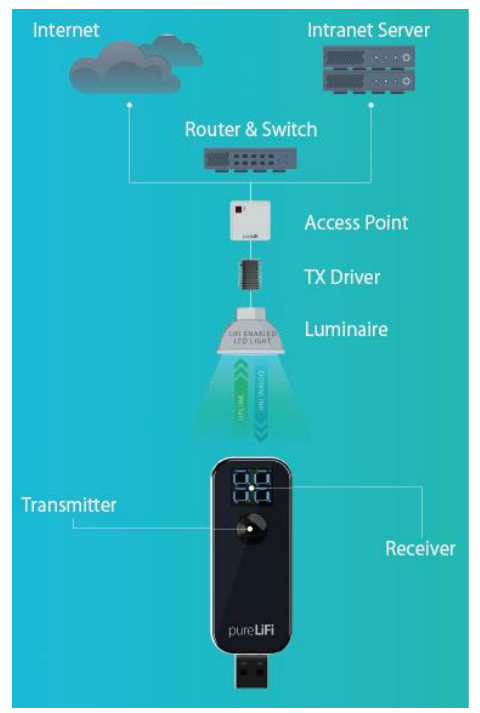

Fuente: Pure Li-Fi

Montaje de Li-Fi: Para el montaje de los equipos Li-Fi se necesita una superficie plana en la que se realizan dos agujeros uno con un diámetro de $65 \mathrm{~mm}$ para el Access Point y el segundo de $182 \mathrm{~mm}$ para la lámpara LED. Ambos agujeros deben tener una separación de máximo 20 $\mathrm{cm}$ medido desde el centro, la figura 5 muestra el montaje general de los equipos para lo cual se construye un cubículo.

Figura 5 Instalación módulos Li-Fi

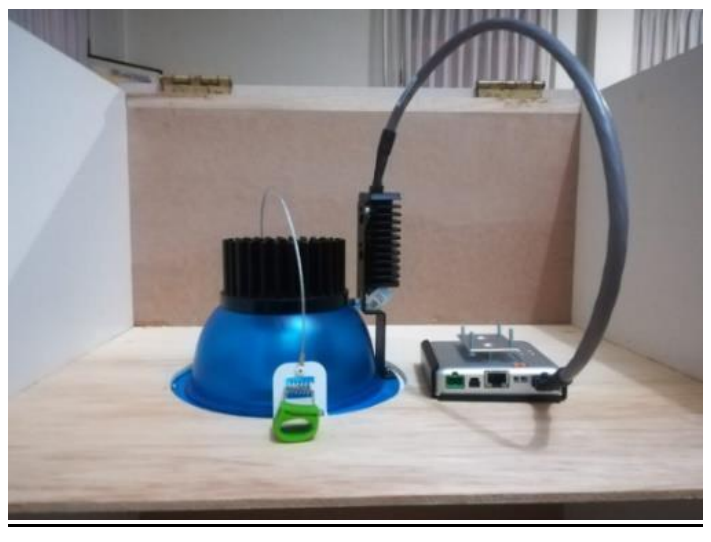

Fuente: Elaboración propia 
Instalación de drivers de Li-Fi: El módulo Li-Fi Xc Station entra en funcionamiento después de instalar en la computadora los drivers para Windows, para lo cual se sigue estos pasos:

1. Dar clic derecho en el archivo install.exe y ejecutar como administrador.

2. Cuando la ventana del instalador del USB Li-Fi-XC se abre, dar clic en siguiente.

3. Dar clic en finalizar en la ventana del instalador del USB Li-Fi-XC.

4. Una vez finalizada la instalación del controlador se conecta el Li-Fi-XC éste crea una red en el computador similar a la red Wi-Fi como se muestra en la figura 6.

Figura 6 Detección de red Li-Fi

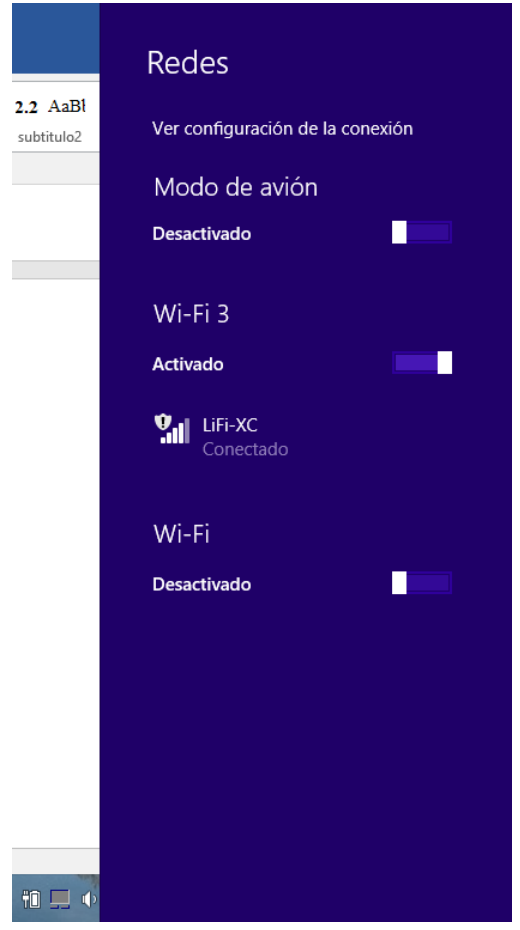

Fuente: Elaboración propia

Es importante conocer si el estado los equipos se encuentran listos para transmitir datos, para ello se debe observar el color del led que tiene cada uno de los dispositivos.

- Rojo: Intenta detectar el controlador.

- Rojo intermitente: falla.

- Naranja: Modo Standby.

- Verde: Encendido y listo para usarse.

b) Conexión y Configuración de la planta EPC

Los algoritmos de control se implementan sobre una planta de laboratorio desarrollada por el fabricante DataLights ${ }^{\circledR}$. Que se muestra en la figura 7. Esta planta de laboratorio permite realizar el control de procesos experimentales de variables como temperatura, velocidad, 
posición y el tratamiento de señales de AC. Ésta contiene el sistema instrumental necesario para la generación y adquisición de datos con una tarjeta basada en microcontroladores; para esto se utilizó la tarjeta MyDAQ que posee $8 \mathrm{I} / \mathrm{O}$ digitales, 3 entradas analógicas y 2 salidas analógicas puras, su resolución en su entrada analógica es de 16 bits, la velocidad de muestreo es de $200 \mathrm{kS} / \mathrm{s}$ (miles de muestras por segundo). También se puede configurar entradas y salidas digitales cuya frecuencia de conmutación es alta para el monitoreo de decodificadores ópticos (encoders) y generación de señales de modulación por ancho de pulso (PWM).

Figura 7 EPC y MyDAQ para los procesos de velocidad y térmico

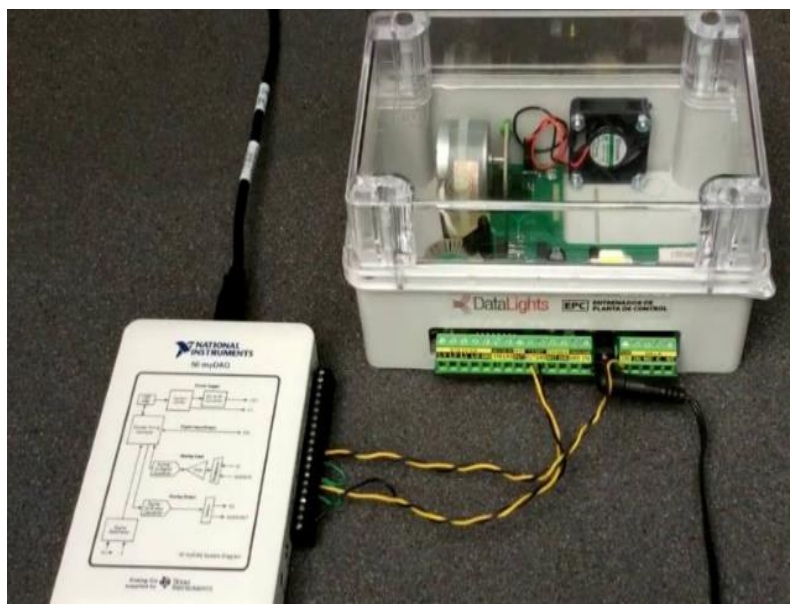

Fuente: Elaboración propia

Desarrollo de Instrumentos Virtuales en LabView2018 (vi): El tiempo de establecimiento de la variable controlada es un dato importante que se debe considerar, por lo tanto, se desea validar la comunicación con dos procesos diferentes en su dinámica. El primero es un proceso cuya dinámica es alta en el cual el actuador principal es un motor de DC cuyo rango de velocidad es de 0 a $4000 \mathrm{rpm}$ y el otro es un proceso con dinámica lenta como es un térmico cuyo rango de funcionamiento oscila entre $0^{\circ} \mathrm{C} \mathrm{a} 80^{\circ} \mathrm{C}$, en este caso el actuador es un diodo led que incrementa su temperatura en función del tiempo de encendido.

Instrumento virtual de velocidad: El desarrollo del instrumento virtual del proceso de velocidad se visualiza en la figura 8 , consta de un ciclo while en cuyo interior se encuentran los iconos del complemento NI DAQmx y Control and Desing Simulation para la adquisición y control de los datos de planta, los cuales son obtenidos por el enconder de manera analógica para ser procesados por el icono Tone Mesurements, el objetivo es conseguir su frecuencia y su amplitud. Si la amplitud es menos que 1 quiere decir que el motor DC no gira, por ende, el encoder entregará una señal sin pulsos, la frecuencia es entonces variable indirecta que se utiliza para medir la variable velocidad. 
Figura 8 Diagrama de bloques del control de velocidad

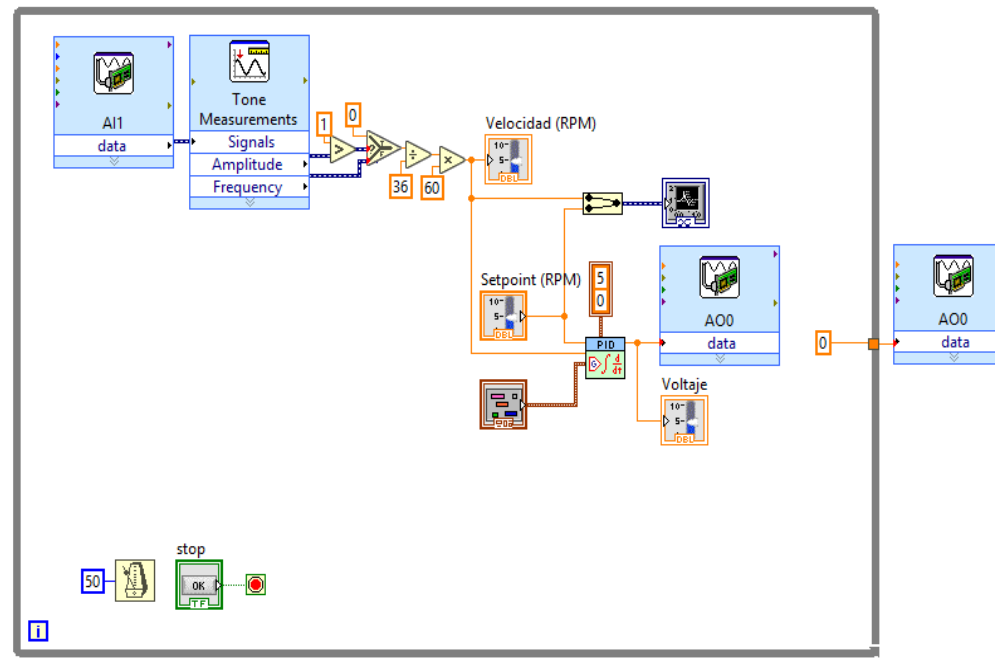

Fuente: Elaboración propia

Instrumento virtual térmico: El desarrollo del instrumento virtual para el control del proceso térmico se ha dividido en dos ciclos while. El primero está relacionado con la adquisición de datos como se puede observar en la figura 9, en el segundo se desarrolla un control del tipo proporcional cuyo actuador térmico es el led y, un control del tipo on/off al ventilador de la planta, también contiene la programación necesaria para incrementar proporcionalmente la temperatura, como se puede observar en la figura 10 que utiliza una señal del tipo PWM para encender de manera proporcional el actuador.

Figura 9 Lazo de adquisición de datos y controlador del proceso térmico

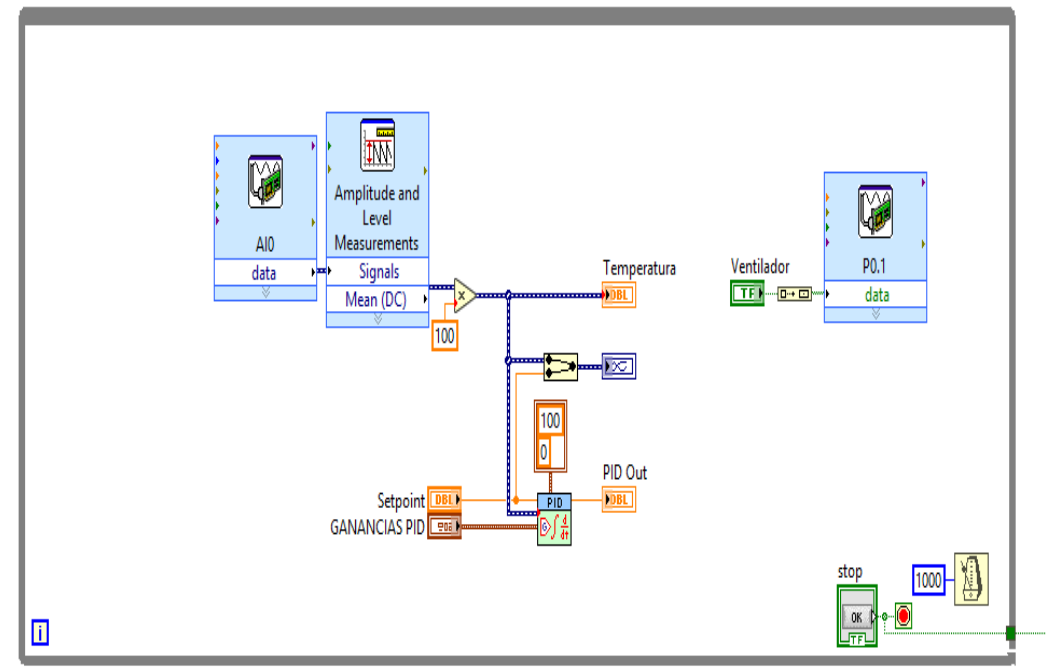

Fuente: Elaboración propia 
Figura 10 Lazo de generación de señal PWM

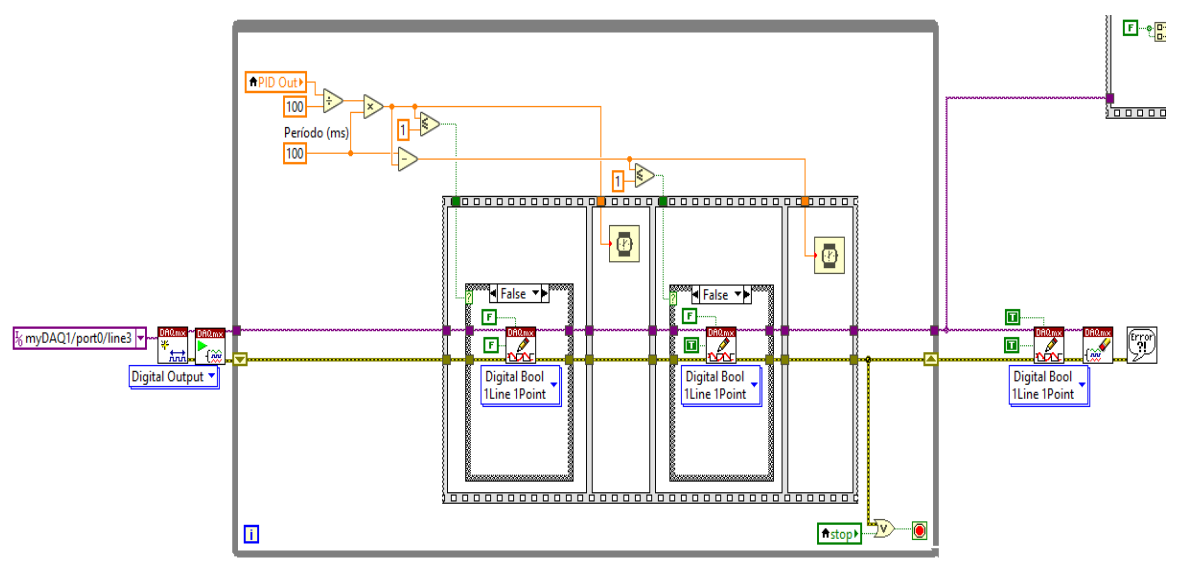

Fuente: Elaboración propia

c) Desarrollo de algoritmos de control

Algoritmo de control de velocidad: Para obtener los valores de los parámetros del controlador se aplica el segundo método de Ziegler-Nichols. Al usar el proceso obtenemos como resultado la velocidad mostrada en la figura 11 la cual nos permite calcular el periodo y determinar las constantes Kcr, Fcr y Pcr detallados en la tabla I. [7]

Figura 11 Señal oscilante para obtener el periodo.

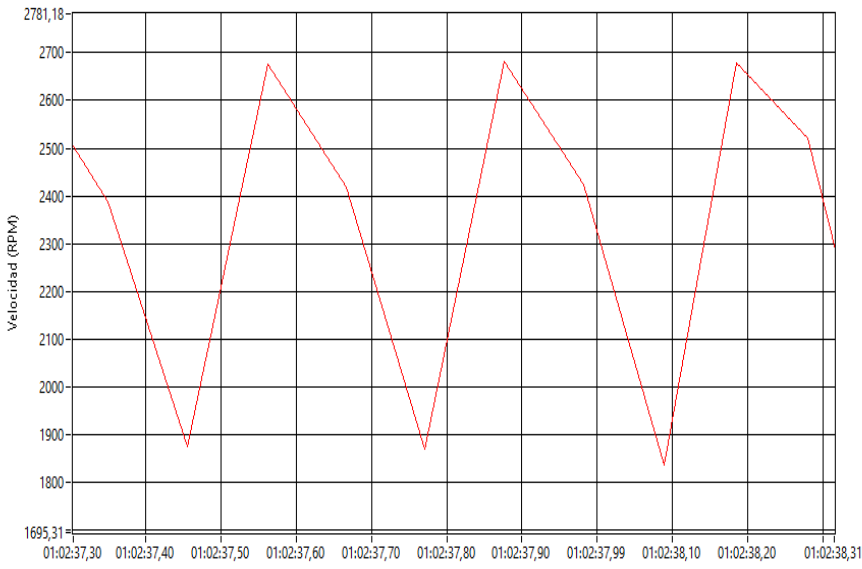

Fuente: Elaboración propia

Tabla 1 Valores de Kcr, Fcr y Pcr usados en la sintonización proceso velocidad

\begin{tabular}{ccc}
\hline PARÁMETRO & VALOR & UNIDAD \\
\hline Kcr & 0.00270 & ------- \\
Fcr & 3.15 & $\mathrm{~Hz}$ \\
Pcr & 0.3467 & $\mathrm{~s}$ \\
\hline
\end{tabular}

Fuente: Elaboración propia 
Algoritmo de control térmico: Para establecer los parámetros del controlador del proceso térmico se utiliza el primer método de Ziegler-Nichols. Con la gráfica generada por el controlador proporcional, se aplica el método experimental de la primera regla de ZieglerNichols [7]. Este consiste en encontrar gráficamente un punto en la respuesta de la planta a un escalón unitario, la curva generada tiene forma de $\mathrm{S}$ y se caracteriza por los parámetros que son: el tiempo de retardo $\mathrm{L}$ y la constante de tiempo $\mathrm{T}$, con estos se obtienen con una recta tangente en el punto de inflexión de la curva y se determina las intersecciones de la recta con el eje del tiempo y el eje de la temperatura como se muestra en la figura 12 y los parámetros conseguidos de manera experimental se detallan en la tabla II.

Figura 12 Respuesta del sistema térmico

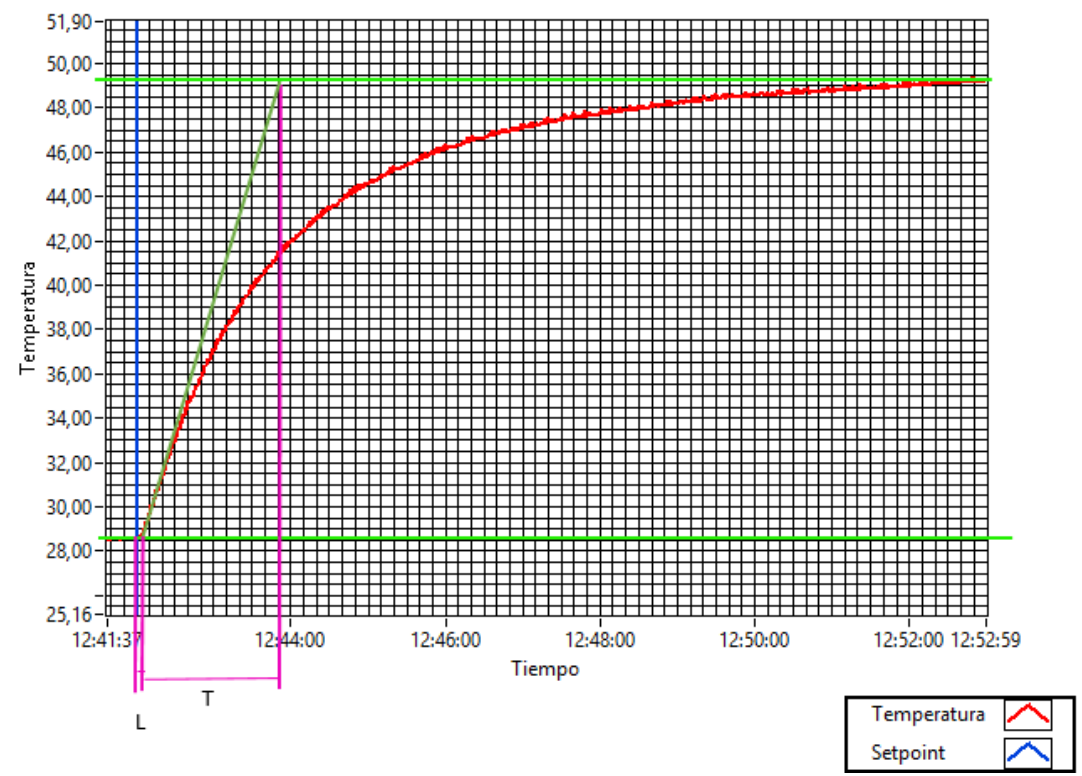

Fuente: Elaboración propia

Tabla 2 Valores obtenidos con el segundo método Z\&N

\begin{tabular}{cccccc}
\hline CONTROLADOR & KC & TI(S) & TI(MIN) & KI(S) & KD(MIN) \\
\hline P & 0.00135 & Inf & Inf & 0 & 0 \\
PI & 0.00121 & 0.26391 & 0.004398 & 0 & 0 \\
PID & 0.00162 & 0.15835 & 0.002639 & 0.03958 & 0.0006598 \\
\hline
\end{tabular}

Fuente: Elaboración propia

Se implementa los tres controladores con los datos obtenidos de los procedimientos de sintonización, como resultado final se obtiene los parámetros expresados en la tabla III, estos controladores se someten a validación. 
Tabla 3 Parámetros de los controladores proceso térmico

\begin{tabular}{cccccc} 
TIPO DE CONTROLADOR & Kc & Ti(s) & Ti(min) & Td(s) & Td(min) \\
\hline P & 21 & inf & inf & 0 & 0 \\
PI & 18.9 & 16.66 & 0.2778 & 0 & 0 \\
PID & 25.2 & 10 & 0.1667 & 2.5 & 0.0417
\end{tabular}

Fuente: Elaboración propia

\section{Resultados}

a) Análisis de desempeño de los controladores.

Controlador de velocidad: Se sintonizó el controlador con los parámetros calculados y mostrados en la tabla I, se probó tres puntos de consigna con los valores 2000, 2500 y 3000 rpm, se calculó el IAE, similar procedimiento se aplica en el algoritmo para el proceso térmico. Para ello se maneja la ecuación 1 , donde $e(t)$ es el igual al punto de consigne menos el valor presente, la ecuación se muestra a continuación: [9]

$$
I A E=\int_{0}^{\infty}|e(t)| d t
$$

Figura 13 IAE de los controladores PI y PID de velocidad

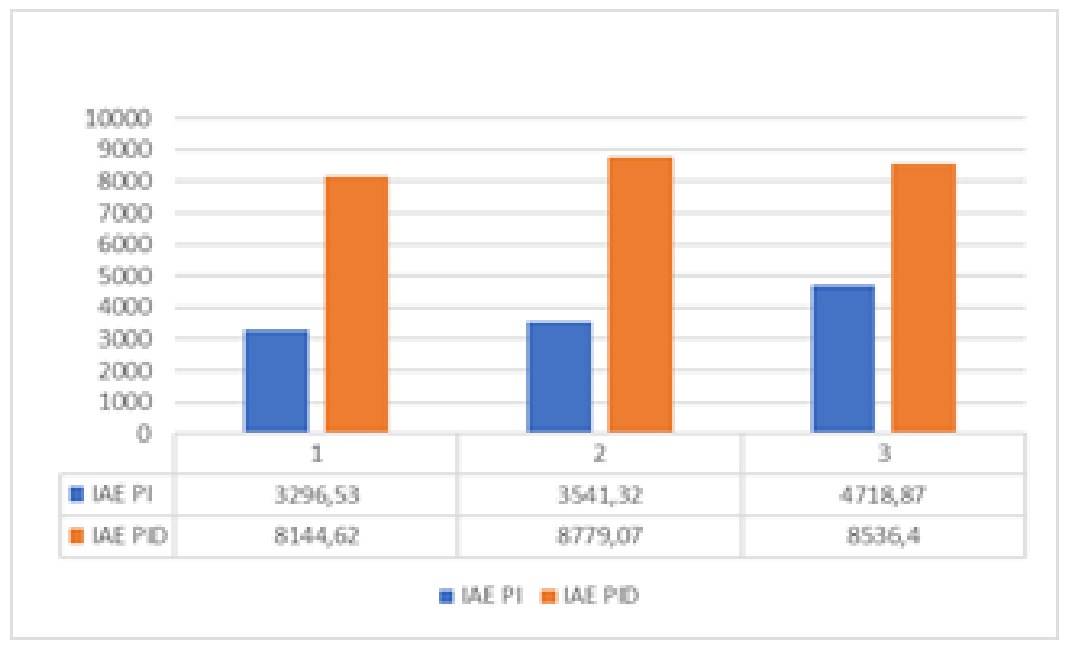

Fuente: Elaboración propia 
Figura 14 IAE de los controladores PI y PID de temperatura con diferentes setpoint

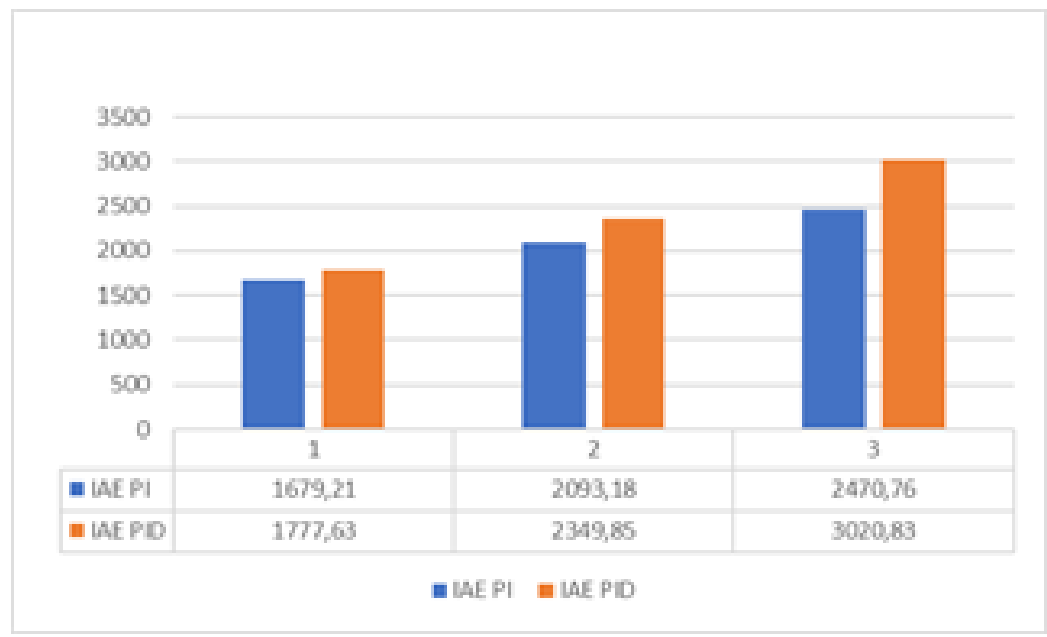

Fuente: Elaboración propia

En los dos casos velocidad y temperatura se analiza el valor final del IAE obtenido con el fin de determinar el algoritmo de control más eficiente en estado estacionario, tanto para el proceso de velocidad como el térmico el algoritmo del tipo PI es el que menor IAE logra en las validaciones.

\section{b) Transmisión inalámbrica}

Una vez determinado el mejor algoritmo de control se desea validar su efectividad a través de la comunicación Li-Fi. Se contrasta con una comunicación del tipo Wi-Fi basada en el protocolo 802.11.n. debido a que es el más usado actualmente y se encuentran en la misma capa del modelo OSI. Es importante examinar la latencia de la comunicación inalámbrica ya que es el vínculo de análisis entre el enlace y los procesos controlados, para esto se utiliza el método de análisis de eficiencia de controladores denominado ITAE (Integral del error absoluto por el tiempo), cuyo cálculo se lo realiza con la ecuación 2.[9]

$$
\operatorname{ITAE}=\int_{0}^{\infty} t|e(t)| d t \quad(2
$$

Proceso de velocidad: En la figura 15 se muestra la respuesta de la planta para el proceso de velocidad con el controlador PI y setpoint de $2000 \mathrm{rpm}$, al observar la figura se determina que el tiempo que necesita el sistema para alcanzar el setpoint establecido de $1 \mathrm{~s}$.

Figura 15 Respuesta del controlador PI con un setpoint de 2000 rpm 


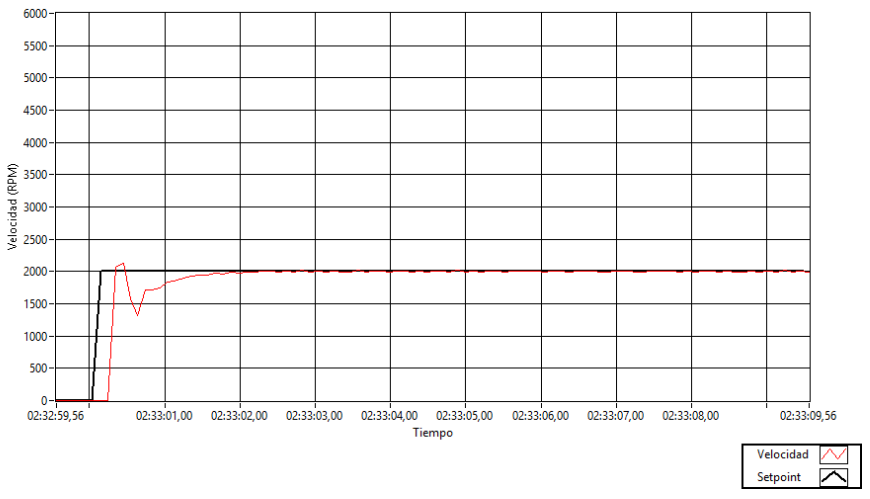

Fuente: Elaboración propia

A través de la herramienta Web Publishing Tool ${ }^{\circledR}$ se recopiló los resultados de la comunicación inalámbrica, tanto para Li-Fi como para Wi-Fi del proceso de velocidad, se divisa los resultados en la tabla IV, además se puede distinguir gráficamente en la figura 16 la diferencia del valor de ITAE para los dos enlaces

Tabla 4 Resultados obtenidos con la evaluación ITAE

\begin{tabular}{ccc}
\hline Setpoint & ITAE Li-Fi & ITAE Wi-Fi \\
\hline $\mathrm{PI} / \mathrm{SP}=2000 \mathrm{rpm}$ & 34272.25 & 218545.68 \\
$\mathrm{PI} / \mathrm{SP}=2500 \mathrm{rpm}$ & 30308.04 & 240984.44 \\
$\mathrm{PI} / \mathrm{SP}=3000 \mathrm{rpm}$ & 31126.96 & 228797.01 \\
\hline
\end{tabular}

Fuente: Elaboración propia

Figura 16 ITAE usando Li-Fi y Wi-Fi en un proceso de velocidad

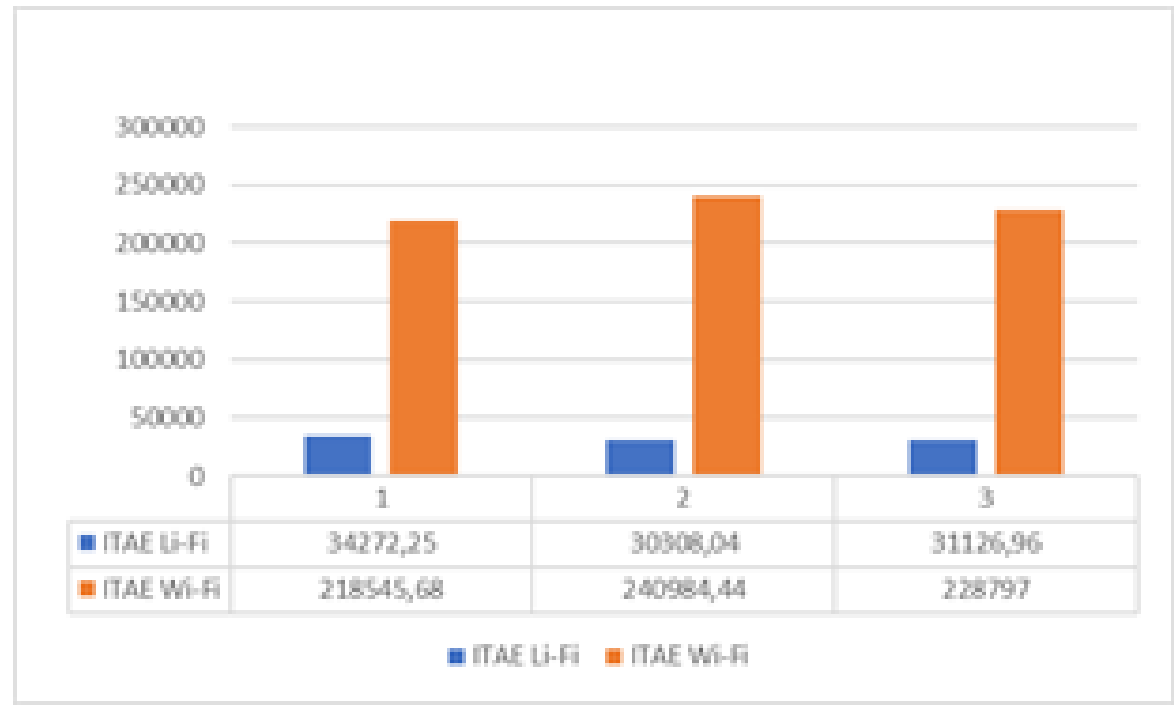

Fuente: Elaboración propia 
Proceso térmico: Con el algoritmo de control del tipo PI se procede a validar su desempeño mediante el método de ITAE se presenta el resultado evaluado con un setpoint de $60^{\circ} \mathrm{C}$, la respuesta del sistema se puede observar en la figura 17. Los resultados del ITAE recopilados a través de Web Publishing Tool son los mostrados en la tabla V

Figura 17 Respuesta del controlador PI del proceso térmico setpoint de $60^{\circ} \mathrm{C}$

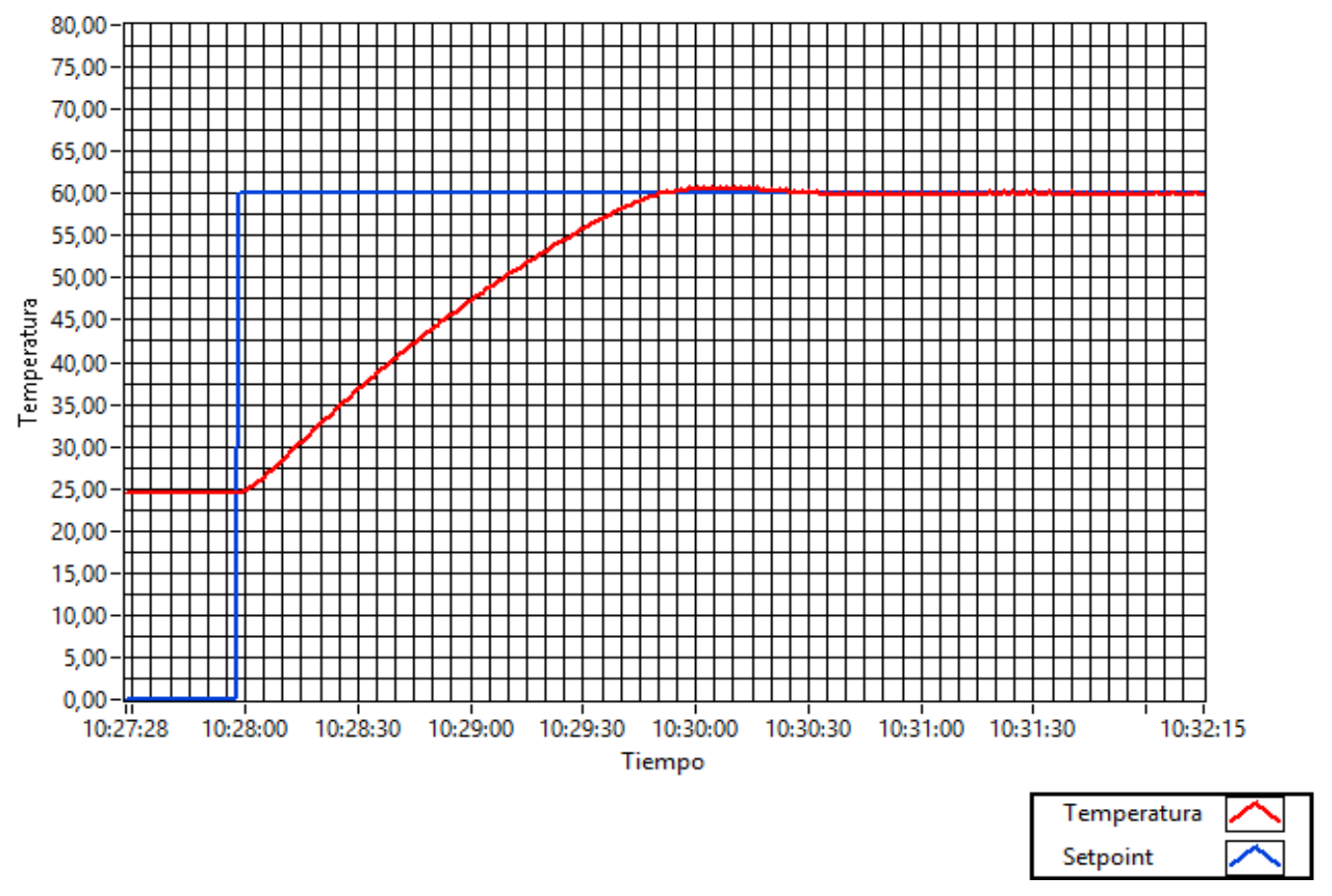

Fuente: Elaboración propia

Tabla 5 Resultados evaluación control de temperatura

\begin{tabular}{ccc}
\hline CTRL/SP & ITAE Li-Fi & ITAE Wi-Fi \\
\hline $\mathrm{PI} / \mathrm{SP}=60{ }^{\circ} \mathrm{C}$ & 1679.21 & 67389.56 \\
$\mathrm{PI} / \mathrm{SP}=65^{\circ} \mathrm{C}$ & 2093.18 & 80989.56 \\
$\mathrm{PI} / \mathrm{SP}=70{ }^{\circ} \mathrm{C}$ & 2470.76 & 82391.62 \\
\hline
\end{tabular}

Fuente: Elaboración propia 
Figura 18 ITAE del proceso térmico comunicación Li-Fi y Wi-Fi

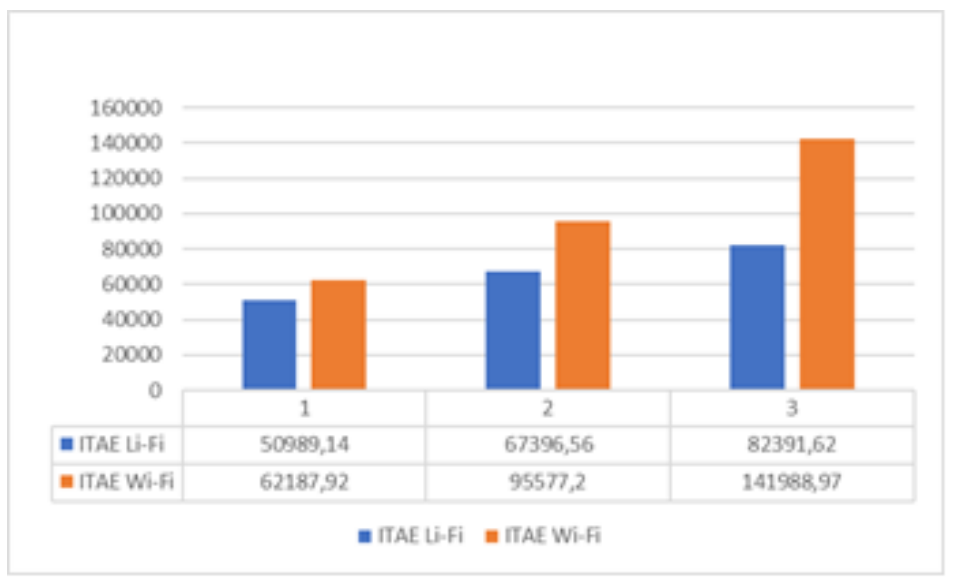

Fuente: Elaboración propia

\section{c) Análisis de desempeño de la comunicación inalámbrica}

Proceso de velocidad: Para la evaluación de la comunicación se realizó el siguiente protocolo de pruebas:

1. Ejecutar el VI del controlador

2. Abrir el programa Wireshark y capturar el tráfico de datos.

3. Monitorear el controlador en la página web

4. Variar el setpoint del controlador.

5. Introducir perturbaciones a la planta de control.

6. Parar la captura de tráfico con Wireshark.

7. Guardar el archivo en formato (.pcapng).

8. Abrir el programa Steelcentral Packet Analyzer e importar el archivo

9. Generar un reporte el cual visualizará el tiempo, los paquetes y bits transmitidos.

En la tabla VI se visualiza los resultados de las comunicaciones inalámbricas aplicadas al proceso de velocidad, la prueba duró 121 segundos para el enlace de Wi-Fi y Li-Fi.

Tabla 6 Comunicación Inalámbrica Li-Fi Y Wi-Fi Proceso Velocidad

\begin{tabular}{ccc}
\hline NOMBRE & Li-Fi & Wi-Fi \\
\hline Número total de bits & 49488 & 102176
\end{tabular}




\begin{tabular}{lrr}
\hline Número total de bytes. & 6186 & 12772 \\
Número total de paquetes & 52 & 102 \\
Número de bytes IP & 5394 & 10452 \\
Número TCP bytes & 3856 & 1102 \\
Número UDP bytes & 1538 & 9078
\end{tabular}

Fuente: Elaboración propia

Figura 19 Bits por segundo y paquetes transmitidos con Li-Fi proceso velocidad

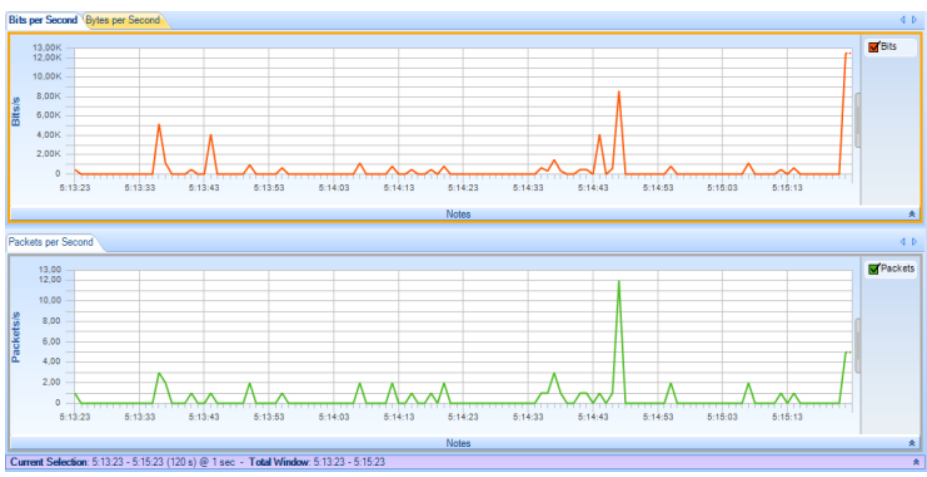

Fuente: Elaboración propia

Figura 20 Bits por segundo y paquetes transmitidos con Wi-Fi proceso velocidad

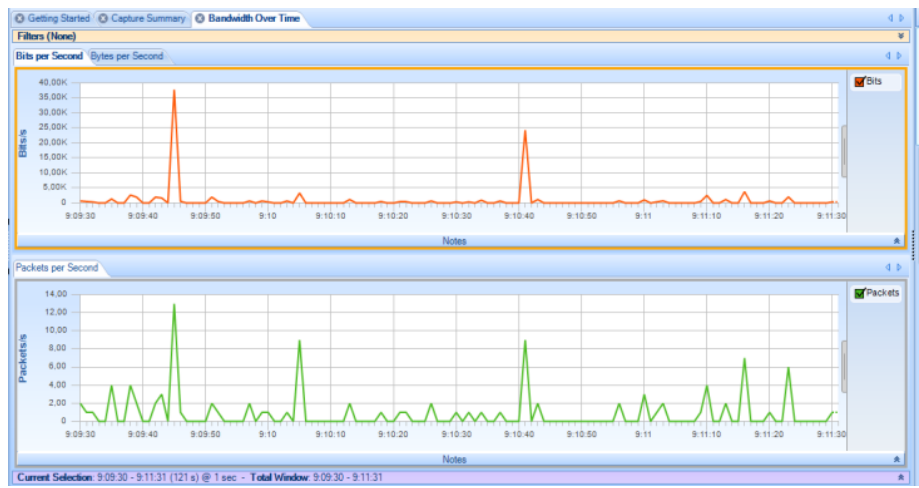

Fuente: Elaboración propia

Proceso térmico: Los resultados de la comunicación inalámbrica se observan en las figuras 21 y 22 además se expresan en la tabla VIII, estos se obtuvieron con el protocolo de pruebas antes mencionado y su duración fue de 854 segundos. 
Tabla 7 Resultados de comunicación Li-Fi para el control de temperatura

\begin{tabular}{lrr}
\hline \multicolumn{1}{c}{ NOMBRE } & Li-Fi & Wi-Fi \\
\hline Número total de bits & 824576 & 1286584 \\
Número total de bytes. & 103072 & 160823 \\
Número total de paquetes & 528 & 813 \\
Número de bytes IP & 92272 & 145503 \\
Número TCP bytes & 75268 & 45380 \\
Número UDP bytes & 17004 & 98213
\end{tabular}

Fuente: Elaboración propia

Figura 21 Bits por segundo y paquetes transmitidos Li-Fi del controlador de temperatura

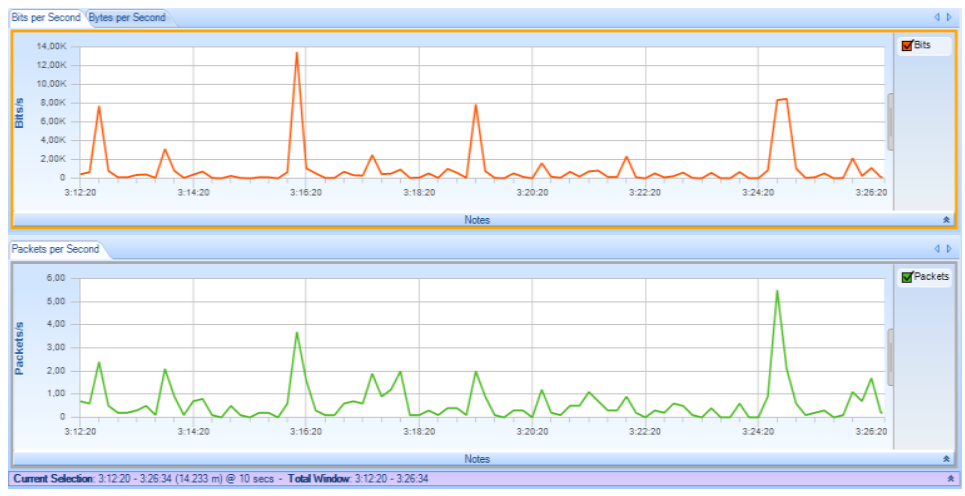

Fuente: Elaboración propia

Figura 22 Bits por segundo y paquetes transmitidos con Wi-Fi del controlador de temperatura

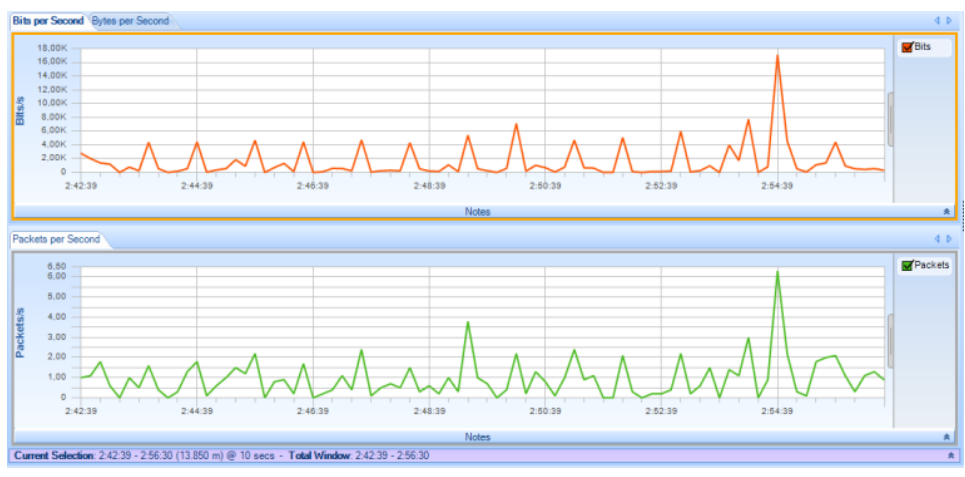

Fuente: Elaboración propia 
Se presenta en las tablas X y XI la condensación de datos comparativos generales de las comunicaciones aplicadas sobre los procesos, los principales valores a tomar en cuenta son la velocidad de transmisión y su latencia.

Tabla 8 Resultados comparativos de comunicación control de velocidad

\begin{tabular}{ccccc}
\hline COMUNICACIÓN & PAQUETES & $\begin{array}{c}\text { BIT } \\
\text { ENVIADOS }\end{array}$ & VELOCIDAD & LATENCIA \\
\hline Li-Fi & 52 & 49488 & $43 \mathrm{Mbps}$ & $1,15 \mathrm{~ms}$ \\
WIFI & 102 & 102176 & $22 \mathrm{Mbps}$ & $4,64 \mathrm{~ms}$ \\
\hline
\end{tabular}

Fuente: Elaboración propia

Tabla 9 Resultados comparativos de comunicación control de temperatura

\begin{tabular}{|c|c|c|}
\hline COMUNICACIÓN & PAQUETES & $\begin{array}{c}\text { BIT } \\
\text { ENVIADOS }\end{array}$ \\
\hline
\end{tabular}

\begin{tabular}{ccccc}
\hline Li-Fi & 528 & 824576 & $43 \mathrm{Mbps}$ & $0.01917 \mathrm{~ms}$ \\
WIFI & 813 & 1286584 & $22 \mathrm{Mbps}$ & $0.05848 \mathrm{~ms}$ \\
\hline
\end{tabular}

Fuente: Elaboración propia

\section{Conclusiones.}

- Los hallazgos de este estudio revelan que las características de los equipos Li-Fi, instalados bajo parámetros establecidos por el fabricante, permitieron desarrollar un sistema de transmisión de datos para un sistema de control de procesos que obtuvo mayor velocidad de transmisión, un menor número de paquetes y un menor tiempo de latencia en la transmisión lo que influye de manera directa sobre el parámetro ITAE como se observa en las tablas X y XI.

- Para Wi-Fi se utilizó el estándar de comunicación punto a punto 802.11n, para una próxima investigación se desea probar la eficiencia de la comunicación Li-Fi con un mayor número de dispositivos conectados, en esta investigación se implementó solo una XC station, no se instaló más de un equipo con estas características debido su costo. 
- Esta investigación fue limitada al entorno en el cual se realizaron sus pruebas, su luminaria proporcionó la máxima cantidad de lúmenes permitidos, se inicia el siguiente trabajo se planteando introducir perturbaciones lumínicas, como las que se puede suscitar en una planta industrial, con el fin de atenuar o incrementar la cantidad de lúmenes proporcionados por el actuador LuciCupLi-Fi, lo que implica diseñar un controlador que permita obtener la menor cantidad de pérdida de información en base a la cantidad de lúmenes emitidos por el actuador

\section{Referencias bibliográficas.}

[1] Espinosa, M., \& Vivanco, M. (2017). Li-Fi: Velocidad de Internet sorprendente bajo la Luz Visible. Killkana Técnica, 1(2), 1-6.

[2] Haas, H., Yin, L., Wang, Y., \& Chen, C. (2015). What is lifi?. Journal of lightwave technology, 34(6), 1533-1544.

[3] Hase, M., Bhanushali, P., Vora, P., Goswami, P., \& Kerawalla, M. A. K. (2016). LIFI-A revolution in the field of wireless-communication. International Journal of Advanced Research in Engineering and Applied Sciences (IJAREAS), 5(4), 10-23.

[4] Ndjiongue, A. R., Ferreira, H. C., \& Ngatched, T. M. (1999). Visible light communications (VLC) technology. Wiley Encyclopedia of Electrical and Electronics Engineering, 1-15.

[5] Núñez, C. V., Peña, J. C., \& Garzón, C. L. (2009). Análisis comparativo de tecnologías inalámbricas para una solución de servicios de telemedicina. Ingeniería y Desarrollo, (25), 200-217.

[6] Páez, M. Á. L., Pineda, J. R., \& Sánchez, E. C. R. (2019). LiFi y su integración con la internet de las cosas. Revista vínculos, 16(1).

[7] Duarte Forero, J. (2015). Optimización de sintonización de controladores tipo PID para procesos térmicos basados en el criterio IAE. INGE@ UAN-Tendencias en la Ingeniería, 5(10).

[8] Swami, N. V. (2015). Li-Fi (light fidelity)-the changing scenario of wireless communication. IJRET: InternationalJournal of Research in Engineering and Technology eISSN, 2319-1163.

[9] Zaldivar, P. M. A., Cingualbres, R. E., Rodríguez, R. P., \& Gutiérrez, A. M. Experimental and numerical evaluation of resilience and toughness in AISI 1015 steel welded plates. Revista Facultad de Ingeniería, (90).

\section{【L Ciencia}




\section{PARA CITAR EL ARTÍCULO INDEXADO.}

Paucar Samaniego, J., Martínez Guashima, O., Guanga Chunata, D., \& Navarrete Chávez, F. (2019). Evaluación de un sistema de comunicación Li-Fi aplicado sobre una planta de control. Ciencia Digital, 3(3.2.1), 88-108. https://doi.org/10.33262/cienciadigital.v3i3.2.1.784

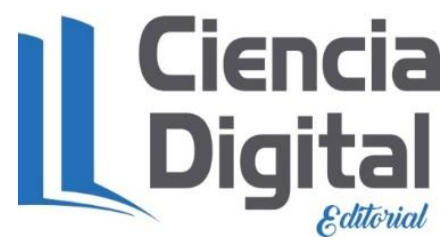

El artículo que se publica es de exclusiva responsabilidad de los autores y no necesariamente reflejan el pensamiento de la Revista Ciencia Digital.

El artículo queda en propiedad de la revista y, por tanto, su publicación parcial y/o total en otro medio tiene que ser autorizado por el director de la Revista Ciencia Digital.
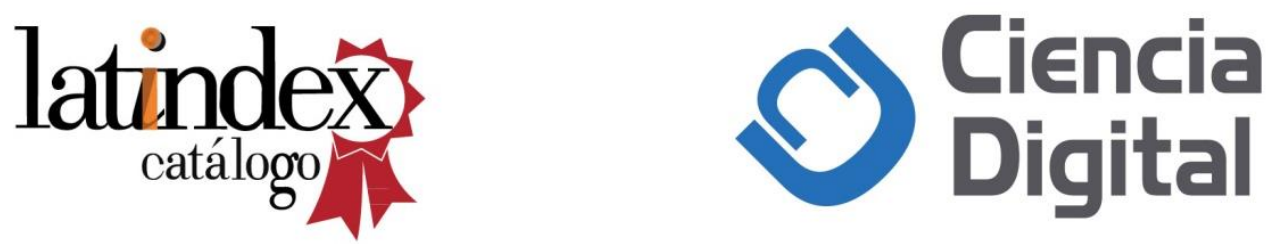